\title{
Voces de estudiantes en clase de geometría y su potencial para desarrollar el discurso en el aula
}

\author{
Students voices in geometry classroom and its potential \\ in the development of classroom discourse
}

\author{
Patricia Perry ${ }^{1}$ \\ Leonor Camargo ${ }^{2}$ \\ Óscar Molina ${ }^{3}$ \\ Carmen Samper ${ }^{4}$
}

\begin{abstract}
Resumen: Presentamos el análisis de expresiones verbales de estudiantes de Educación Básica secundaria, en interacción comunicativa en una clase de geometría sobre semejanza de triángulos, usando rasgos propios del discurso matemático. Adoptamos una estrategia investigativa "basada en prácticas usuales", con el objetivo de contrastar la hipótesis que teníamos, según la cual las verbalizaciones de los estudiantes en el aula de matemáticas suelen no ser útiles para el desarrollo del respectivo discurso, debido a rasgos intrínsecos de esas intervenciones. La hipótesis no se sostiene pues encontramos voces de estudiantes que tienen un potencial considerable para impulsar el discurso. Sin embargo, no siempre son aprovechadas por el profesor, quizá debido a
\end{abstract}

Fecha de recepción: 08 de noviembre de 2019. Fecha de aceptación: 16 de enero de 2021.

1 CIUP, Universidad Pedagógica Nacional, Bogotá, Colombia, pperryc@yahoo.com.mx, orcid.org/00000002-9162-071X

2 Departamento de Matemáticas, Facultad de Ciencia y Tecnología, Universidad Pedagógica Nacional, Bogotá, Colombia, Icamargo@pedagogica.edu.co, orcid.org/0000-0002-2237-7306

3 Departamento de Matemáticas, Facultad de Ciencia y Tecnología, Universidad Pedagógica Nacional, Bogotá, Colombia, ojmolina@pedagogica.edu.co, orcid.org/0000-0003-4797-1173

4 Departamento de Matemáticas, Facultad de Ciencia y Tecnología, Universidad Pedagógica Nacional, Bogotá, Colombia, csamper@pedagogica.edu.co, orcid.org/0000-0003-2342-8950 
una gestión que tiene como prioridad lograr la intervención de los estudiantes a costa de ejercer su papel de líder del discurso.

Palabras clave: voz matemática del estudiante, discurso matemático, enfoque comunicacional

\begin{abstract}
We present, using features of mathematical discourse, an analysis of eighth grade, Middle School, students' verbal expressions, found in the communicative interaction that took place during a geometry class about similar triangles. We adopt a research strategy "based on usual practices", with the objective of contrasting the hypothesis we had: students' verbal utterances in a mathematics class are not usually useful for the development of the respective discourse, due to intrinsic features of those interventions. Our hypothesis does not hold because we found student voices that have considerable potential to boost mathematical discourse. However, these are not always taken advantage of by the teacher, perhaps due to a classroom management that prioritizes achieving student intervention at the cost of exercising the role of discourse leader.
\end{abstract}

Keywords: student mathematical voice, mathematical discourse, communicational approach

\title{
1. INTRODUCCIÓN
}

Las tendencias curriculares actuales promulgan que la enseñanza debe proporcionar oportunidades para dar sentido a las matemáticas a través del discurso (NCTM, 1989; MEN, 1998); aceptan que aprender matemáticas es una actividad sociocultural; y le apuestan a que el discurso en el aula mejora la comprensión compartida y el nuevo discernimiento. En ese marco, el desarrollo de la Educación en Colombia, en los últimos 25 años, ha influido para que la enseñanza tradicional en el aula (i.e., exposición del profesor y ejercitación del estudiante), descrita en la literatura (e.g., Gregg, 1995; Gates y Vistro-Yu, 2003), haya dado paso a una en la que, grosso modo, el desarrollo de los temas se hace mediado por tareas asignadas a los estudiantes, cuyas respuestas se exponen ante todo el grupo en intercambios principalmente orales. Así, en la actualidad no es raro encontrar aulas de matemáticas en las que los estudiantes hablan entre ellos y 
con el profesor sobre asuntos matemáticos implicados en las tareas que realizan (e.g., Forero-Sáenz, 2008; Jiménez et al., 2010; Sfard, 2015). Frente a esta realidad, cabe preguntarse si las contribuciones de los estudiantes desempeñan o pueden desempeñar un papel relevante en el aprendizaje de las matemáticas en el aula.

En calidad de hipótesis inicial, en un proyecto desarrollado en $2018^{5}$, formulamos nuestro problema de investigación así: rara vez las intervenciones de los estudiantes en clase de matemáticas del nivel de educación básica y media son realmente útiles para avanzar en el desarrollo del tema matemático que se estudia pues suelen exponer ideas incompletas, confusas y no articuladas al intercambio verbal en el que se presentan. Nuestro objetivo investigativo fue contrastar tal hipótesis examinando dinámicas discursivas en diversas clases de geometría. Buscamos lo que denominamos voces de estudiantes, argumentamos por qué las consideramos voces, describimos algunos de sus rasgos a la luz del enfoque que propone Sfard (2008a) y examinamos su potencial para avanzar en el aprendizaje.

En este artículo presentamos ejemplos de expresiones verbales de estudiantes de 12-14 años, surgidas en un episodio de interacción comunicativa cuando el profesor les pidió determinar si dos polígonos particulares dados son semejantes. Estas voces son ejemplos de la evidencia que nos permite afirmar que nuestra hipótesis no se sostiene tal cual, pues son expresiones que sí tienen un potencial considerable para impulsar el discurso. Sugerimos que, quizá debido a que la gestión del profesor prioriza la amplia participación de los estudiantes sobre su papel como líder del discurso, no siempre las voces son aprovechadas por el profesor.

\section{MARCO TEÓRICO}

La fundamentación conceptual del estudio proviene del campo investigativo Lenguaje en la Educación Matemática y particularmente de la línea "matemáticas como discurso y discurso matemático" (Radford y Barwell, 2016), con foco en expresiones de los estudiantes surgidas en interacciones comunicativas en el aula. Diversos trabajos se enmarcan en la línea mencionada. Por ejemplo: Clarke et al. (2013) se interesan por las ocasiones que tienen los estudiantes de

5 El proyecto de investigación, Voces de los estudiantes en la clase de geometría, fue desarrollado por el grupo Aprendizaje y Enseñanza de la Geometría de la Universidad Pedagógica Nacional (Colombia). 
escuchar y decir términos matemáticos; Esmonde y Langer-Osuna (2013) y Jung y Shütte (2018) se centran en la búsqueda de estrategias de enseñanza para favorecer la participación discursiva de los estudiantes en condiciones de equidad; Kaur (2013) e Ingram et al. (2019) examinan la expresión pública de los estudiantes, buscando que ellos compartan sus pensamientos con sus compañeros con apoyo del profesor o autónomamente. En nuestro trabajo nos centramos en rasgos discursivos de la comunicación de contenidos matemáticos específicos, línea en la que se ubica la elaboración teórica seminal de la investigadora Anna Sfard.

\subsection{DISCURSO MATEMÁTICO Y APRENDER MATEMÁTICAS}

En el enfoque que propone Sfard, la comunicación está en el corazón de la educación dado que "la necesidad de comunicación es la primera fuerza conductora tras todos los procesos cognitivos humanos" (Sfard, 2008b, p. 71), y el pensamiento se conceptualiza como una forma de comunicación con uno mismo (Sfard, 2008b). Aquí "comunicación" refiere al uso y producción de recursos con la intención de que un interlocutor, que puede ser el mismo hablante, reaccione de una cierta manera (Sfard, 2008c).

Entendemos que los actos comunicativos son "intentos para comprometer a los miembros de una comunidad en actividades indispensables para satisfacer las necesidades humanas, desde las más primitivas y fundamentales para sobrevivir hasta las necesidades culturales más avanzadas y complejas" (Sfard, 2008b p. 77). Así, es natural que distintas comunidades tengan distintas maneras de comunicarse. Sfard denomina discurso a una forma diferenciada de comunicación que agrupa a algunos individuos y deja por fuera a otros. Cualquier sociedad humana se puede dividir en comunidades de discurso que se solapan parcialmente. La membrecía a una comunidad de discurso se gana participando en actividades de comunicación de cualquier colectivo que practique ese discurso, sin importar qué tan pequeño sea (Sfard, 2008d).

En nuestro estudio, la comunidad de discurso matemático está conformada por profesor, estudiantes del curso en cuestión y observadores externos no participantes que estuvieron presentes en las clases durante las que se desarrolló la temática polígonos semejantes; el discurso matemático de la clase está constituido por las actividades de comunicación en esta comunidad, en el espacio y tiempo de la observación. 
Haciendo eco a Sfard (2014), vemos las matemáticas como una actividad de contar cierto tipo de relatos y, por tanto, como un discurso, distinguible de otros mediante cuatro rasgos externos: vocabulario, mediadores visuales, rutinas y narrativas aceptadas. Además, entendemos que aprender matemáticas es "el proceso de cambiar de cierta manera, bien definida, las formas discursivas propias" (Sfard, 2008e, p. 44). Esto implica, por una parte, ampliar el vocabulario, construir nuevas rutinas y producir nuevas narrativas aceptadas, y, por otra parte, hacer cambios en las reglas sobre el discurso. Significa que "algunas tareas familiares como, por ejemplo, definir una palabra o identificar figuras geométricas, se harán ahora de una manera diferente, no familiar" (Sfard, 2008d, p. 256).

Enseguida, unas palabras sobre los rasgos externos del discurso matemático escolar:

Vocabulario y su uso: refiere principalmente a las palabras clave -en geometría, por ejemplo, las relativas a formas de objetos geométricos y a relaciones entre estos- y al uso de ellas de acuerdo con las definiciones dadas o las reglas dictadas por la respectiva comunidad de discurso matemático, en este caso, la escolar. Algunas palabras clave del discurso matemático son exclusivas de este (e.g., "homotecia"), y otras hacen parte del repertorio de discursos coloquiales (e.g., "semejanza"); en el segundo caso, aprender requerirá pasar del uso cotidiano al uso especializado. Tal como lo señala Sfard (2008d), el uso del vocabulario es un asunto muy importante porque, siendo equivalente a lo que otros llaman 'significado del término', es responsable de lo que el usuario puede decir sobre (y ver en) el mundo.

Mediador visual y su uso: es un objeto visible sobre el que se opera y que hace parte integral del acto de comunicar y de los procesos de pensamiento; no es un mero recurso auxiliar para expresar el pensamiento preexistente (Sfard, 2007). Es un medio con el que quien participa en un discurso matemático identifica el objeto de su conversación y coordina su comunicación (e.g., las etiquetas literales asignadas a los vértices de un polígono son mediadores visuales para referirse a un ángulo o lado particular del polígono). Los mediadores visuales especializados tienen formas de uso especializadas (e.g., para referir, eliminando ambigüedad, a un determinado ángulo, la etiqueta del vértice debe nombrarse entre las otras dos letras que identifican un punto en cada lado).

Rutina: "una rutina realizada en una situación de tarea dada por una persona dada es la tarea, como la entiende el realizador, junto con el procedimiento que siguió para realizar la tarea" (Lavie, Stein y Sfard, 2019, p. 161). Una situación de tarea refiere a un escenario en el que una persona se siente obligada a hacer 
algo; puede estar creada, intencionalmente o no, como invitación a una cierta clase de acción. Una tarea como la entiende el realizador es el conjunto de todos los rasgos característicos de eventos precedentes que la persona considera que requieren replicación. Un procedimiento implementado por el realizador de una tarea en respuesta a una situación de tarea dada es la prescripción para la acción que se ajusta a la realización actual y también a aquellas en las que está modelado, es decir, no es una réplica perfecta de la acción precedente sino una réplica selectiva -preserva unos aspectos de la realización anterior y cambia otros-.

Narrativa: es cualquier secuencia de verbalizaciones, escrita u oral, que describe un objeto, establece una relación entre objetos o narra un proceso relativo a objetos, y está sujeta a aceptación o rechazo con la ayuda de procedimientos de justificación específicos del discurso; una narrativa aceptada se etiqueta usualmente como verdadera (Sfard, 2008d). En el discurso matemático especializado, es habitual presentar las narrativas aceptadas como si se refirieran a entidades que existen en el mundo, de manera objetiva, e independiente de la acción humana; este proceso conocido como objetificación incluye dos movimientos discursivos estrechamente relacionados, que ocurren por separado: reificación y alienación (Sfard, 2008d). "La reificación es el acto de remplazar oraciones sobre procesos y acciones con proposiciones sobre estados y objetos" (Sfard, 2008d, p. 44); esto implica remplazar verbos por sustantivos. La alienación es el acto de "usar formas discursivas que presentan los fenómenos de una manera impersonal, como si ocurrieran por sí mismos, sin la participación del ser humano" (Sfard, 2008d, p. 295). Por ejemplo, un relato impersonal sobre el procedimiento seguido para determinar si se cumplen las condiciones de la definición de polígonos semejantes para un caso particular ejemplificaría una narrativa no objetificada por no ser reificada. En el mismo contexto, "La semejanza de esos dos polígonos es indecidible" es un ejemplo de narrativa objetificada (con el sustantivo "semejanza" se habla de una relación entre dos objetos) y alienada (es una oración atemporal en la que no hay asomo de la agencia humana).

\subsection{VOZ MATEMÁTICA DEL ESTUDIANTE}

Aquí nos enfocamos en las verbalizaciones de los estudiantes que consideramos voz matemática, debido al papel importante que desempeñan o pueden desempeñar en el desarrollo del discurso matemático del aula. Denominamos voz matemática del estudiante (en adelante, voz del estudiante) a la expresión oral, 
escrita o gestual, auténtica e inteligible, emitida por el estudiante en interacción con el profesor u otros estudiantes, para comunicarse (con la intención ${ }^{6}$ de exponer una idea, presentar una inquietud, preguntar, objetar, etc.) sobre un asunto matemático acerca del cual versa la clase. El adjetivo "auténtica" pretende destacar el carácter no impostado de la expresión, que permite reconocerla como "propia" de quien la emite y en la que se identifica un compromiso personal. El adjetivo "inteligible" quiere aclarar que la expresión es interpretable por quien la recibe.

\subsection{DEFINICIÓN DE POLÍGONOS SEMEJANTES Y UNO DE SUS USOS}

Un análisis de la definición dada ${ }^{7}$ en la clase puede aportar elementos para el análisis de las voces que aquí se consideran. La definición enuncia que:

"Dos polígonos convexos son semejantes si y solamente si existe una correspondencia biunívoca entre sus vértices, tal que:

los ángulos correspondientes tienen igual medida,

las razones entre las longitudes de los lados correspondientes son iguales."

La narrativa, expresada en calidad de definición, plantea un enunciado bicondicional mediante el uso del término lógico "si y solamente si".

Así, para alguna correspondencia biunívoca entre vértices de dos polígonos convexos, los dos enunciados condicionales refieren a relaciones recíprocas de dependencia entre la relación geométrica "ser semejantes" (en pares de polígonos convexos) y la conjunción de otras dos relaciones geométricas: "tener la misma medida" (en pares de ángulos correspondientes) y "ser iguales" (en pares de razones de medidas de longitud de lados correspondientes). Por tanto, los dos enunciados condicionales permiten respaldar dos procedimientos matemáticos, respectivamente: uno, dados dos polígonos convexos semejantes, determinar los atributos que tienen los ángulos y los lados correspondientes de los dos polígonos; dos, dados dos polígonos convexos, inferir si son semejantes

\footnotetext{
6 Las intenciones comunicativas pocas veces se hacen explícitas, pero usualmente son rastreables mediante pistas comunicativas en el contexto de una interacción.

7 Nos ceñimos a la definición dada por el profesor, dejando de lado la formulación que preferimos (e.g., la condición relativa a los ángulos, la formularíamos así: las medidas de amplitud de los ángulos correspondientes son iguales).
} 
examinando si los atributos de sus ángulos y lados correspondientes tienen las condiciones suficientes para ello.

Esta narrativa objetificada mediante la cual comienza a precisarse el uso estándar del término polígonos convexos semejantes, para la comunidad de discurso de nuestra aula, incluye varios vocablos especializados para los que el profesor no hizo explícita mención de cómo deberían entenderse. Entre ellos destacamos tres. (i) "Polígono convexo", término que refiere a los polígonos para los cuales todos sus vértices, excepto los dos que determinan cualquiera de sus lados, pertenecen al mismo semiplano respecto a la recta que contiene el lado mencionado; (ii) "correspondencia biunívoca entre vértices de dos polígonos", término que refiere a un conjunto de pares ordenados de vértices de los polígonos, en los que cada vértice de uno de los polígonos (el primero que se considera) tiene asociado uno y solo un vértice del otro polígono; (iii) "razón entre longitudes", término que refiere al cociente de dos medidas de longitud.

Respecto al enunciado condicional que permite decidir si dos polígonos son semejantes, cabe esbozar dos asuntos que están implicados y, por tanto, hacen parte fundamental de "desempacar" (Selden y Selden, 1995) la narrativa: (i) la correspondencia debe ser biunívoca, por tanto los dos polígonos deben tener el mismo número de vértices; (ii) existen muchas correspondencias biunívocas posibles entre los vértices de los dos polígonos y se debe escoger una que parezca útil para lo que se quiere; en este caso, una que forme pares de lados y ángulos con el mismo atributo cualitativo relativo en los dos polígonos (e.g., el par de lados más largos, el par de lados más cortos, el par de ángulos con igual amplitud, etc.).

\subsection{RASGOS DISCURSIVOS DE NUESTRA RESPUESTA A LA SITUACIÓN DE TAREA PROPUESTA}

\subsubsection{Nuestra rutina y algo más}

La situación de tarea propuesta por el profesor incluye dos cuadriláteros no convexos; pregunta si se podría decir que son semejantes atendiendo a las dos características que da la definición. La tarea, tal como la entendemos, nos pone dos requerimientos estrechamente relacionados pero distinguibles: (i) usar la definición como guía para establecer un plan de acción para abordar la pregunta y (ii) implementar el procedimiento puntual para responder la pregunta. En ese sentido y con el propósito de tener un referente amplio para analizar producciones 
de los estudiantes, presentamos un plan de acción (columna izquierda, Cuadro 1) y los procedimientos genéricos asociados (columna derecha, Cuadro 1).

Cuadro 1. Plan de acción y procedimientos genéricos para resolver la situación de tarea

\begin{tabular}{|c|c|c|}
\hline & $\begin{array}{c}\text { Plan de acción genérico para abordar la } \\
\text { pregunta }\end{array}$ & $\begin{array}{c}\text { Procedimiento genérico para responder la } \\
\text { pregunta }\end{array}$ \\
\hline Paso 1 & $\begin{array}{l}\text { Decidir si el caso particular es un elemento } \\
\text { del conjunto de objetos geométricos a los } \\
\text { que alude la definición. Si no lo es, el uso } \\
\text { de la definición no es pertinente; la res- } \\
\text { puesta es algo como: "No ha lugar", termi- } \\
\text { nando así el plan. Pero si lo es, procedemos } \\
\text { con el Paso } 2 \text {. }\end{array}$ & $\begin{array}{l}\text { Examinar si son polígonos convexos, usan- } \\
\text { do la respectiva definición. }\end{array}$ \\
\hline Paso 2 & $\begin{array}{l}\text { Decidir si hay alguna correspondencia biu- } \\
\text { nívoca entre los vértices de los dos polígo- } \\
\text { nos que sea una buena candidata para } \\
\text { elegir. Si no se encuentra tal corresponden- } \\
\text { cia, un argumento centrado en el objeto } \\
\text { matemático "formas diferentes" es suficiente } \\
\text { para concluir que los polígonos dados no } \\
\text { son semejantes, y aquí terminaría el plan. Si } \\
\text { se encuentra, procedemos con el Paso } 3 \text {. }\end{array}$ & $\begin{array}{l}\text { Determinar si los polígonos tienen el mis- } \\
\text { mo número de vértices. Si es así, con base } \\
\text { en recursos visuales, poner en relación la- } \\
\text { dos y ángulos de las dos figuras de mane- } \\
\text { ra que se identifique (si la hay) una } \\
\text { correspondencia útil para examinar la se- } \\
\text { mejanza. }\end{array}$ \\
\hline Paso 3 & $\begin{array}{l}\text { Decidir si uno de los dos atributos (i.e., relativo } \\
\text { a los lados o a los ángulos), se cumple para } \\
\text { el caso particular. Si no se cumple, es sufi- } \\
\text { ciente un argumento que atienda al esque- } \\
\text { ma lógico "negación de una conjunción" (i.e., } \\
\text { no se cumple la igualdad de las razones o } \\
\text { no se cumple la igualdad de las medidas de } \\
\text { los ángulos) para concluir que no son seme- } \\
\text { jantes, terminando aquí el plan. De lo contra- } \\
\text { rio, procedemos con el Paso } 4 \text {. }\end{array}$ & $\begin{array}{l}\text { Determinar, por ejemplo, si las medidas de } \\
\text { amplitud de los ángulos correspondientes } \\
\text { son iguales o no, con base en una compa- } \\
\text { ración de cantidades numéricas. }\end{array}$ \\
\hline Paso 4 & $\begin{array}{l}\text { Decidir si el otro atributo se cumple. Si no se } \\
\text { cumple, la conclusión es la no semejanza de } \\
\text { los dos polígonos en cuestión, y si se cumple, } \\
\text { la conclusión es la semejanza de aquellos. }\end{array}$ & $\begin{array}{l}\text { Determinar las razones de las medidas de } \\
\text { longitud de los lados correspondientes y } \\
\text { verificar si son o no iguales. }\end{array}$ \\
\hline
\end{tabular}

Nota: Si no se tiene información sobre las medidas de amplitud de los ángulos o de la longitud de los lados, la conclusión inmediata es que no se puede determinar si son o no semejantes. 


\subsubsection{Vocabulario}

Hay varios términos especializados involucrados al resolver la situación de tarea. En primer lugar, "ángulos correspondientes" y "lados correspondientes". En ambos casos, el uso pertinente de los términos se da con referencia a dos polígonos cuyos vértices se han puesto en correspondencia biunívoca. En el caso de la segunda condición, encontramos dos términos especializados: "Iongitudes de los lados correspondientes" y "razones entre las longitudes de los lados correspondientes", términos que describen gradualmente el sujeto gramatical del cual se predica en el enunciado. Haciendo eco a Duval (2017/2004, p. 139), vemos que el encadenamiento de complementos del sustantivo "lados" es un indicador del lenguaje matemático especializado, que contrasta con lo usual en la lengua natural donde la operación de descripción rara vez va más allá de usar un solo complemento del sustantivo (e.g., razones de los lados).

\subsubsection{Mediadores visuales}

La imagen icónica de los cuadriláteros (Imagen 1) que hace parte de la situación de tarea constituye el mediador visual obligado por cuanto expone el objeto al que refiere la pregunta y al que debe referir la respuesta. De este mediador visual cabe destacar dos rasgos que pueden influir considerablemente en el uso que le den los estudiantes. (i) Ambas figuras tienen la misma posición relativa con respecto al marco donde se presentan, situación que sugiere una cierta correspondencia entre los vértices de los dos cuadriláteros y, por tanto, induce a su uso con base en la mera percepción inmediata. Sin desconocer el papel imprescindible de la percepción en la elección de una correspondencia entre vértices, existe un procedimiento que no depende de la posición, que atiende al número de vértices de cada figura y a la determinación de pares de lados y de ángulos con el mismo atributo cualitativo relativo en los dos polígonos. (ii) Las letras con las que se designan los vértices de los polígonos tienen un tamaño muy reducido, condición que dificulta su uso por parte de los estudiantes cuando hablan desde sus puestos. Otros posibles mediadores visuales útiles son las marcas distintivas en ángulos de las figuras para señalar elementos asociados por la correspondencia que se identifica como la candidata más adecuada para examinar las condiciones de la definición. 
Imagen 1. Cuadriláteros incluidos en la situación de tarea
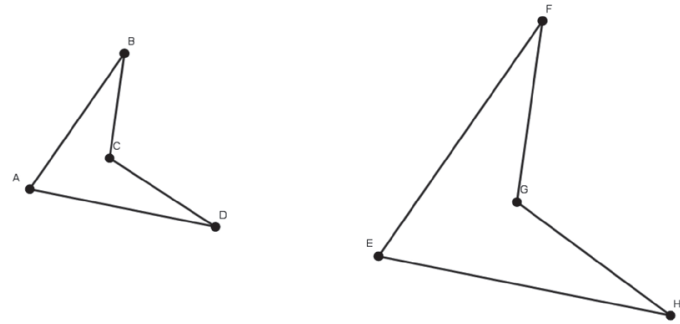

\subsubsection{Narrativa}

Nuestra narrativa de respuesta a la situación de tarea es: "No es posible determinar si los polígonos dados son semejantes usando la definición, porque no son convexos y además sin las medidas de amplitud de ángulos y de longitud de lados no es posible establecer si las igualdades a las que alude la definición se cumplen o no". Es una narrativa objetificada en la que el objeto es el procedimiento para determinar si dos polígonos dados son semejantes, del cual se predica su no aplicabilidad para el caso particular dado; incluye además un argumento mediante el cual la narrativa se puede aceptar como verdadera, dado que obedece al manejo lógico de una proposición condicional como garantía: si la condición suficiente no se tiene, no es posible tener una conclusión respecto a la condición necesaria.

\section{ASPECTOS METODOLÓGICOS}

Acorde con el propósito de la investigación se adoptó una estrategia investigativa "basada en prácticas usuales" (Camargo, en prensa). La información analizada en este artículo proviene de una sesión de clase de geometría en un curso de grado séptimo de un colegio de Bogotá, conformado por 35 estudiantes, 20 hombres y 15 mujeres con edades entre 12 y 14 años.

Registramos en video la interacción comunicativa durante la sesión de clase del 11 de mayo de 2018. También hicimos grabaciones de audio. Hicimos la transcripción a partir de los archivos de audio y la complementamos con las grabaciones de video, logrando textos alimentados con fotografías y aclaraciones recogidas en notas de campo. 
Obtuvimos los datos investigativos del estudio haciendo una adaptación de la ruta sugerida por Villalta (2009). Comenzamos por segmentar la transcripción correspondiente a la clase, en términos de los intercambios verbales públicos ocurridos durante la clase, sobre ideas tratadas como parte de la conversación. Teniendo esta información, construimos episodios investigativos, reuniendo todos los intercambios en los que la idea tratada hace parte de un tema de conversación. El Episodio 2, Dos polígonos dados, ¿se podría decir que son semejantes, usando las dos características de la definición?, del cual provienen las expresiones verbales que analizaremos, reúne 10 intercambios en los que los estudiantes responden o hacen comentarios relacionados con el tema de conversación. Por esta vía identificamos las expresiones susceptibles de ser voz matemática.

Teniendo en mente el propósito del estudio construimos dos conjuntos de rasgos: uno para decidir si las expresiones previamente seleccionadas podían ser declaradas voz matemática; otro para describir las voces atendiendo a rasgos distintivos del discurso matemático, para lo cual tuvimos en cuenta propuestas de Tang et al. (2012), Morgan y Sfard (2016), y Wang (2016). En el Cuadro 2 y el Cuadro 3 presentamos descripciones de los rasgos.

Cuadro 2: Rasgos de la expresión verbal que la caracterizan como voz matemática

\begin{tabular}{|c|c|}
\hline Rasgo & Descripción \\
\hline Inteligibilidad & Verbalización susceptible de ser interpretada de manera sustentable. \\
\hline Autenticidad & $\begin{array}{l}\text { Verbalización que manifiesta lo que el estudiante piensa, cree, opina, sabe, etc. } \\
\text { Es una expresión genuina suya y no una mera repetición de una frase hecha } \\
\text { por otro. (Circunstancias que indican autenticidad: ser el primero en manifes- } \\
\text { tar una idea o un punto de vista respecto a lo dicho por otro, aclararlo, am- } \\
\text { pliarlo o corregirlo). }\end{array}$ \\
\hline
\end{tabular}


Cuadro 3: Rasgos para describir la voz matemática

\begin{tabular}{|c|c|}
\hline Rasgo & Descripción \\
\hline \multicolumn{2}{|l|}{ Vocabulario y uso } \\
\hline Especialización & $\begin{array}{l}\text { Incluye pertinentemente palabras y frases especializadas del discurso mate- } \\
\text { mático. } \\
\text { Usa léxico especializado acorde con el empleo que dicta la comunidad del } \\
\text { discurso de referencia. } \\
\text { Usa la respectiva sintaxis en la construcción de narrativas (i.e., orden y com- } \\
\text { binación de las palabras para expresar significados). }\end{array}$ \\
\hline $\begin{array}{l}\text { Complejidad } \\
\text { lógica }\end{array}$ & $\begin{array}{l}\text { Usa conectivos lógicos de manera acorde con las reglas de la lógica. } \\
\text { Usa cuantificadores de manera acorde con las reglas de la lógica. }\end{array}$ \\
\hline \multicolumn{2}{|c|}{ Mediadores visuales y su uso } \\
\hline \multirow[t]{4}{*}{ Especialización } & Crea o usa recursos gráficos para: \\
\hline & $\begin{array}{l}\text { 1. Reconocer directamente o identificar propiedades visuales (e.g., formas } \\
\text { geométricas bidimensionales, amplitud de ángulos, posiciones de las lí- } \\
\text { neas, orientación física de una figura). }\end{array}$ \\
\hline & $\begin{array}{l}\text { 2. Identificar condiciones necesarias y suficientes de una definición: figuras, } \\
\text { rectas auxiliares, ángulos. }\end{array}$ \\
\hline & $\begin{array}{l}\text { Usa notación simbólica o diagramas para indicar relaciones de dependencia } \\
\text { entre propiedades. }\end{array}$ \\
\hline \multicolumn{2}{|l|}{ Rutinas } \\
\hline $\begin{array}{l}\text { Manera de } \\
\text { hacer }\end{array}$ & $\begin{array}{l}\text { Lleva a cabo un procedimiento (conjunto de una o más acciones realizadas } \\
\text { en un cierto orden) o algunas de sus acciones. } \\
\text { Reconoce un propósito y/o una tarea asociados al procedimiento. }\end{array}$ \\
\hline \multicolumn{2}{|l|}{ Narrativas } \\
\hline De qué hablan & $\begin{array}{l}\text { Habla de un objeto o de una relación entre objetos (emplea sustantivo). } \\
\text { Habla de un proceso (emplea verbo). }\end{array}$ \\
\hline Respaldo & $\begin{array}{l}\text { Provee argumentos acordes con las reglas del discurso matemático (narrati- } \\
\text { va respaldada). } \\
\text { Provee argumentos informales (inducción, abducción, analogía) para apoyar } \\
\text { una aserción. }\end{array}$ \\
\hline $\begin{array}{l}\text { Hacia la } \\
\text { reificación }\end{array}$ & $\begin{array}{l}\text { Reformula enunciado sobre proceso (en términos de acciones) como enun- } \\
\text { ciado sobre objeto o sus propiedades (en términos de sustantivos). }\end{array}$ \\
\hline Alienación & $\begin{array}{l}\text { Formula verbalización en la que la agencia humana está opacada (forma } \\
\text { impersonal e intemporal). }\end{array}$ \\
\hline
\end{tabular}




\section{CONTEXTO EN EL QUE SURGIERON LAS EXPRESIONES ANALIZADAS}

La clase en la que surgieron las expresiones analizadas aquí fue la cuarta de una secuencia didáctica implementada a través de siete sesiones. Se desarrolló en torno al uso de la definición de polígonos semejantes para determinar si parejas de polígonos eran semejantes. Salvo en dos episodios, en los cuales el tema es una pregunta o un comentario hechos por un estudiante, fue el profesor quien planteó el asunto de conversación, mediante una pregunta, formulada oralmente y por escrito. Los estudiantes respondieron o reaccionaron oralmente, a veces apoyándose en el registro gráfico, en turnos asignados por el profesor, que no necesariamente alternaban la voz del profesor y la de un estudiante.

La reacción del profesor a lo expuesto frecuentemente se redujo a una palabra que indicaba haber escuchado lo dicho, seguida por la acción de dar la palabra a otro estudiante o emitir una verbalización que sugería o indicaba cambio del asunto de conversación. En algunos casos, después de escuchar a un estudiante, sugirió sutilmente un aspecto específico para seguir la conversación y lo hizo generalmente parafraseando o repitiendo algo de lo que se acababa de verbalizar. Tras escuchar varias respuestas, el profesor, con algún criterio que no siempre fue evidente para nosotros, dio por terminada una conversación para iniciar otra en torno a otra situación de tarea. La escasa realimentación ofrecida por el profesor llegó a exasperar a algunos estudiantes, quienes le pedían que precisara lo correcto o, por lo menos, que les dijera si lo expresado por un estudiante particular era correcto o no. Pero el profesor se mantuvo en su posición de no evaluar ni realimentar las intervenciones de los estudiantes.

Del que hemos Ilamado Episodio 2, presentamos el análisis de expresiones de cuatro estudiantes, elegidas por su riqueza para ilustrar los elementos considerados en el análisis, que se articulan en una conversación y permiten evidenciar por qué conducen a cuestionar nuestra hipótesis inicial, aunque no llegaran a jugar un papel evidente y relevante en el avance del discurso matemático del aula.

\section{ANÁLISIS DE EXPRESIONES VERBALES DE ESTUDIANTES}

En el tablero aparece proyectada la definición de polígonos semejantes dada por el profesor. Al final de un intercambio verbal entre el profesor y algunos 
estudiantes, a petición del profesor, $\mathrm{JJ}^{8}$ expresa "las dos" condiciones para que dos polígonos sean semejantes: "los ángulos siempre tienen que ser iguales" y "las razones entre sus lados también tienen que ser iguales". Enseguida el profesor plantea al curso la situación de tarea, constituida por la imagen gráfica de dos cuadriláteros no convexos (Imagen 1) y la pregunta "Si yo atiendo a esas dos características que dice JJ, ¿̇yo podría decir que estas dos figuras son semejantes?".

\subsection{NO, PORQUE NO SE PUEDE AFIRMAR QUE LOS ÁNGULOS SEAN IGUALES}

Voz de JN (interviene a solicitud del profesor)

Sí. (En voz baja) [66] Creo que sí. [68] Porque... son iguales... (...) (Se sonríe) [70] La figura pequeña... pues... [72] [Las dos figuras son iguales] [p]ues, no en tamaño sino en forma. [74] $]^{9}$

De esta expresión vamos a analizar la oración "LLas dos figuras son iguales] no en tamaño sino en forma". Las cuatro verbalizaciones anteriores -surgidas en interacción con el profesor, quien le formuló preguntas buscando precisión o ampliación de lo que acababa de decir (i.e., “¿Sí o no?", "¿Por qué?", “¿Qué son iguales?", “¿Son iguales las dos figuras?")- nos permiten contextualizar y dar sentido completo a la última oración. En consecuencia, la mencionada expresión nos resulta inteligible.

En su expresión, bien formada desde el punto de vista gramatical, JN, de manera dubitativa, responde afirmativamente la pregunta inicial del profesor y argumenta recurriendo a la forma de las figuras que observa en la proyección. Al ser la primera respuesta y una que no se ciñe completamente a la solicitud del profesor pero que es pertinente, además de estar formulada en un estilo cercano al lenguaje coloquial, consideramos que es voz.

En cuanto al vocabulario y su uso, todos los términos presentes en la oración son palabras clave del discurso geométrico. Es notorio que en lugar de "polígono" usa el término "figura" y que no articula la expresión "figuras semejantes".

\footnotetext{
8 Remplazamos los nombres de los estudiantes por dos letras: JJ, JN, JH DG, SF, SG.

9 En la transcripción usamos: ( ) para incluir descripción de acciones o detalles del contexto; (...), (......) o (... ......) para indicar silencio más o menos largo en la verbalización; "palabra"... para indicar un pare en lo que se estaba diciendo; [numeral] para indicar el puesto de la verbalización de la que fue tomada o parafraseada la cita; [ ] para incluir comentario que ayude a la claridad de la verbalización.
} 
Emplea los términos "tamaño" y "forma" para referir a dos atributos propios de las figuras. El uso que da a la frase "son iguales en forma" es el que se da en el lenguaje coloquial para significar un parecido notable. En suma, no podemos decir que el uso de todos los términos especializados que incluye se ajuste bien a lo que dictan las matemáticas escolares.

Aunque tácito, es claro que el sujeto de la oración refiere a las figuras dadas en la situación de tarea; así, la narrativa de JN habla de objetos y predica sobre dos rasgos de aquellos. Es decir, reconocemos una narrativa objetificada que se puede someter a consideración para determinar si se acepta o rechaza. Para justificar por qué cree que las figuras son semejantes, JN presenta una descripción, resultado de una comparación holística, no detallada, de las dos figuras que, de hecho, son los mediadores visuales en la comunicación; esa manera de reconocer que "son iguales en forma" está basada exclusivamente en su percepción visual inmediata. Es una rutina usual en la cotidianidad para reconocer, por ejemplo, caras en fotografías a diferentes escalas, que se distancia de la solicitud del profesor de considerar las dos condiciones de la definición de semejanza de polígonos.

En reacción a la voz de JN, el profesor destacó el uso de la palabra "forma". Reconocemos en la voz de JN una contribución potencialmente productiva para el desarrollo del discurso matemático del aula, en dos sentidos. Por una parte, si se abordaran preguntas tendientes a precisar qué es la "forma de un polígono" y qué debe cumplirse para que dos polígonos sean iguales en forma, se podría abrir una vía para reificar el proceso de comparar formas de polígonos en el objeto polígonos semejantes. Por otra parte, si se abordara la pregunta "itienen la misma forma?" en diversos casos de pares de polígonos para los que no se dan sus medidas se podría generar una oportunidad para comenzar a ver la necesidad y utilidad de contar con un criterio común (que podría ser sugerido por la definición) para responder esa pregunta de manera informada.

\section{Voz de JH}

(Desde su puesto) Es que los ángulos son iguales en las dos figuras. [84] El que está en la prime, en la figura pequeña eee... hay uno abierto y tres cerrados [86] (Pasa al tablero) Este ángulo... (hace una marca en torno al vértice del ángulo que parece recto en el polígono pequeño) es igual a este (hace una marca en torno al vértice del ángulo que parece recto en el otro polígono), solo que el tamaño de la figura pues cambia. [88] 
Imagen 2. Usa mediador visual para indicar los objetos a los que se refiere
Imagen 3. Modifica el mediador visual usado

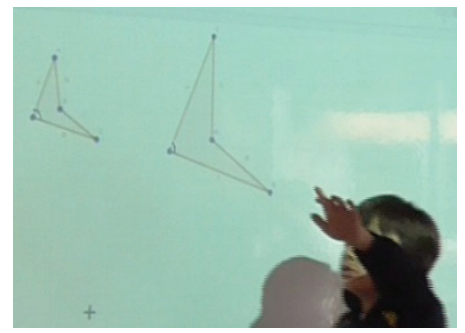

Esta expresión de JH es voz. Surge, de manera voluntaria y en respuesta a la situación de tarea planteada. Para su análisis, tomamos las tres intervenciones hechas en turnos distintos como un todo -sabiendo que entre ellas media una solicitud del profesor buscando precisión (i.e., "¿Cuáles ángulos?", "Pase al tablero a mostrar los ángulos iguales")- con el propósito de tener una respuesta lo más completa posible.

En su expresión, bien formada desde el punto de vista gramatical, JH particulariza la condición relativa a los ángulos de los polígonos a los polígonos expuestos en el tablero. Primero, afirma que los ángulos son iguales, luego se refiriere a los ángulos de una de las figuras mediante atributos y, finalmente, afirma que dos ángulos específicos, uno de cada figura, son iguales. Aunque reconocemos imprecisiones en esta expresión, la interpretación que acabamos de darle nos la hace inteligible. También la consideramos auténtica pues es la primera en la que se intenta atender una de las dos condiciones de la definición y, sobre todo, es la primera en la que de manera explícita se alude a rasgos de los ángulos que podrían servir para elegir una correspondencia entre vértices de los dos polígonos, asunto este que no se ha mencionado en manera alguna hasta el momento. ${ }^{10}$

Acerca del vocabulario y su uso, todos los términos presentes en la expresión de JH son palabras del discurso geométrico. De nuevo, es notorio que en lugar de "polígono" usa el término "figura" y que no articula la expresión "figuras

10 Podría pensarse que la oración inicial de JH es mera repetición de lo dicho previamente por JJ (i.e., "los ángulos siempre tienen que ser iguales"). La diferencia en la forma verbal de las dos oraciones (i.e., "tienen que ser" y "son") nos lleva a ver diferencias: en el primer caso, es una prescripción, en el segundo es una descripción. 
semejantes". Emplea el término "tamaño" para referir a un atributo de las figuras y manifiesta que "el tamaño de la figura cambia" en lugar de decir, por ejemplo, "las figuras no tienen el mismo tamaño", probablemente influido por la experiencia del uso de la opción Homotecia en GeoGebra. Usa también el término "ángulo", pero no, "ángulos correspondientes"; en cambio, acuña dos términos relacionados, "ángulo abierto" y "ángulo cerrado", que, al parecer, pretende emplear para insinuar un criterio para determinar la correspondencia de ángulos de "la figura pequeña" con ángulos de la otra figura. Los adjetivos "abierto" y "cerrado" parecen referirse al atributo amplitud de los ángulos. Finalmente, con el término "igual" predica una relación entre objetos -i.e., ángulos- para significar, como es usual en las matemáticas escolares y en la lengua natural- una relación relativa a un determinado atributo (i.e., amplitud de ángulo) o una relación relativa a la medida de ese atributo (i.e., medida de amplitud de ángulo); claramente, no pretende decir que los ángulos son el mismo como debería entenderse desde un punto de vista rigurosamente matemático. En suma, no podemos decir que el uso de todos los términos especializados que incluye se ajuste bien a lo que dictan las matemáticas escolares, pero no es tan desajustado como para no poder entender lo que pretende decir.

Aunque JH no usa el término "ángulos correspondientes", cuando puede actuar directamente sobre las figuras expuestas en el tablero, se vale de mediadores visuales -trazos curvos- (Imagen 2 e Imagen 3) para indicar, a la vez que usa el deíctico "este", los dos ángulos de los que habla, que efectivamente parecen estar en una correspondencia conveniente para lo que interesa.

Enfocándonos en la segunda oración de JH, cuando hace una descripción de los ángulos de la figura pequeña, vemos que comienza a esbozar un criterio (i.e., que el ángulo sea abierto o cerrado) para determinar una correspondencia que le permita identificar los ángulos de los cuales afirma que son iguales. Así, parece ser que JH está comenzando a generar una rutina de búsqueda de ángulos correspondientes de interés para la semejanza, que no se limita a la percepción visual inmediata.

En la tercera oración de JH, "Este ángulo... es igual a este, solo que el tamaño de la figura pues cambia", reconocemos una narrativa sobre dos ángulos, cada uno de los cuales hace parte de una de las figuras expuestas en el tablero, de los que se predica su igualdad. Es decir, reconocemos una narrativa objetificada que se puede someter a consideración para determinar si se acepta o rechaza.

El profesor aprovechó la voz de JH para preguntar a otro estudiante si estaba de acuerdo en la "igualdad de esos ángulos" y, por esa vía, el estudiante llegó a afirmar 
que no se podría asegurar, sin una herramienta para medir, que los ángulos "son iguales", y que por ello no se podría asegurar que las figuras son semejantes.

Reconocemos en la voz de JH una contribución potencialmente productiva para el desarrollo del discurso matemático del aula en lo concerniente a la identificación de una correspondencia entre vértices de los polígonos, a la luz de la cual se examinan las dos condiciones que menciona la definición.

\subsection{SI SE CUMPLIERA LA SEGUNDA CONDICIÓN, ¿SE PODRÍA DECIR QUE SON SEMEJANTES?}

Aunque podía parecer que la situación de tarea estaba resuelta, la intervención de DG muestra que no para todos era así.

Voz de DG

Haciendo lo que dice aquí... (señala su cuaderno) queeee... midiendo las longitudes [107] y viendo la razón de ellas, entonces eeeee... el que está acá... (ademán con los brazos) de la figura pequeña... [109] No, no alcanzo a ver. Bueno, ese con este (ademán que indica pasar a la otra figura) y dividirlos, y si..., y así con todos los demás (ademán para aludir a las dos figuras), y si da lo mismo, ¿̇significaría que... sí es semejante? [111]

La expresión espontánea de DG es voz. Nos resulta inteligible: el estudiante pregunta si bajo el supuesto de que la segunda condición se cumpliera para el caso de las figuras expuestas, eso significaría que una de ellas es semejante a la otra. También, la consideramos auténtica: DG es el primero en considerar la condición relativa a los lados, pero no para examinarla en el caso que tienen entre manos pues él propone una situación hipotética al respecto, sino para cuestionar la suficiencia de la segunda condición para concluir que los dos polígonos son semejantes. Ello enfoca la manera de usar la definición de polígonos semejantes en el caso en cuestión.

DG usa un vocabulario que incluye varios términos propios del discurso de la semejanza de polígonos, concernientes, específicamente a la condición relativa a los lados. Usa "razón" para referirse a la división de medidas de longitud; no usa término alguno para indicar las parejas de medidas que conforman la razón; para ello se vale de deícticos y señalamientos sobre la figura que actúan como mediador visual. Usa "figura" en lugar de "polígono". Usa el cuantificador 
universal (i.e., "y así con todos los demás") para incluir los cocientes entre medidas de todas las parejas de lados correspondientes. El uso de los términos especializados que incluye parece estar en concordancia con el sugerido por las matemáticas escolares.

Enfocando la atención en la situación hipotética que DG plantea, reconocemos una narrativa no objetificada en la que el estudiante describe el procedimiento que podría convertirse en rutina para examinar si la segunda condición de la definición se cumple (i.e., medir longitudes, determinar medidas que va a dividir, dividir medidas, comparar resultados) y su pregunta va encaminada a especificar el criterio de decisión respecto a la semejanza o no de los polígonos.

En reacción a la voz de DG, el profesor emitió un "ok". Reconocemos en la pregunta de DG una contribución potencialmente productiva para el desarrollo del discurso matemático del aula. Abordarla, recurriendo a considerar casos particulares en los que sea posible contrastar las condiciones suficientes para asegurar la semejanza de los polígonos con las que permiten afirmar la no semejanza, podría contribuir no solo a profundizar en la significación de la definición de polígonos semejantes, sino también a avanzar en el uso de los conectivos lógicos "y" y "o" y la negación de estos, según lo dictan las reglas de la lógica.

\section{3 ¿CÓMO PROCEDER PARA EXAMINAR LO RELATIVO A LA PRIMERA CONDICIÓN?}

Después de la intervención de DG, hay dos intercambios en los que, de manera difusa, JH insinúa una relación entre homotecia y lados proporcionales con una relación multiplicativa y JJ, una relación entre homotecia y ángulos congruentes. El profesor aclara que no saben si se utilizó homotecia y que lo único que tienen son las figuras dadas; luego, repite que para asegurar que dos figuras sean semejantes se requiere asegurar que "los ángulos son iguales y que las razones entre los lados son iguales". Enseguida SF dice: "Pero si uno no tiene un transportador a la mano, ¿cómo hace?". El profesor repite textualmente las palabras de SF y SG reacciona esbozando una idea incompleta en la que sugiere el uso de la homotecia.

Voz de SG

Me parece que, leyendo la guía, por ahí, me pareció ver que... digamos, si tú trazas una línea desde la parte superior de la figura ya agrandada (desde su puesto señala hacia el tablero) hasta la misma de la chiquita [130] (pasa al tablero a petición 
del profesor) No sé si me acuerdo bien, pero en la guía, él [el autor] hacía una línea como desde acá (comienza a trazar una línea recta desde uno de los vértices del polígono más grande hasta uno del otro polígono y la extiende un poco) y otra desde acá, algo así (acción similar a la anterior para otro par de vértices), y luego cuando se juntaban aquí (marca el punto de intersección, Imagen 4a) entonces la figura como que se... (desde muy cerca del vértice del ángulo, desplaza hacia la derecha la mano abierta como barriendo la región angular determinada por las líneas trazadas, y cambia ligeramente su orientación con respecto al tablero, Imagen 4b) se agrandaba de acá a acá (desde la posición de la figura pequeña, desplaza hacia la derecha la mano extendida perpendicular al tablero y paralela a uno de los lados de la figura pequeña, Imagen 4c) [134].

Imagen 4. Gestos con los que SG acompañó su relato usando como mediador visual el dibujo realizado en el tablero

a

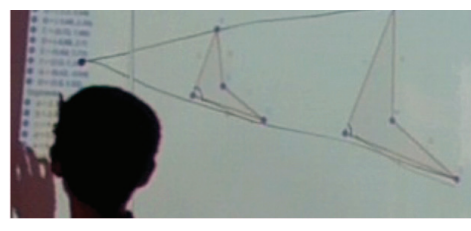

b
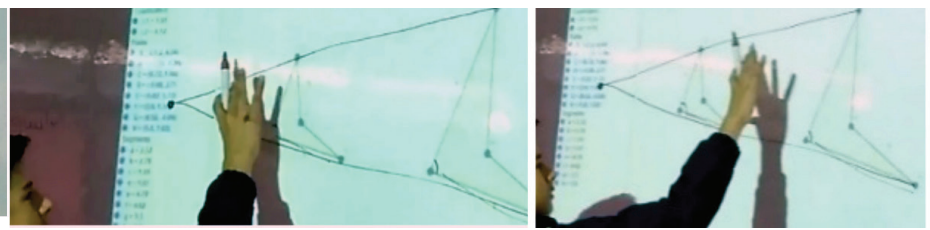

d
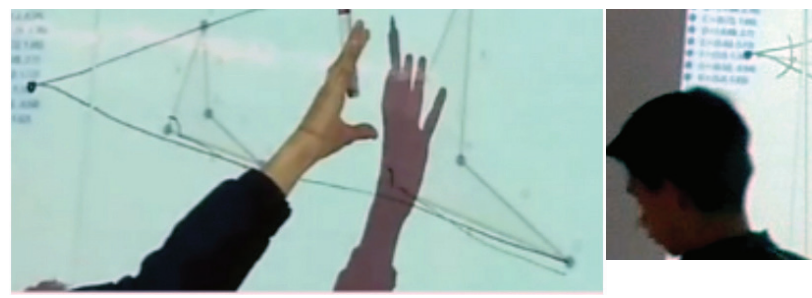

(Por sugerencia del profesor, SG tiene en cuenta otro de los vértices del cuadrilátero pequeño) iAh! Creo que ahí, esta sería como que así (traza la línea a la que pertenecen la intersección de las otras dos, y otro par de vértices que podrían ser correspondientes), y digamos este ángulo (hace dos marcas, que trazadas parecen una sola, para referirse al ángulo determinado por las primeras dos líneas trazadas, ver Imagen 4d), tal vez, algo tendría que ver. (......) No me acuerdo de más. (Vuelve a su puesto) [141]. 
Para el análisis, reunimos en una sola expresión las intervenciones de SG, teniendo en cuenta que la última es reacción a una sugerencia que le hizo el profesor.

La expresión de SG, surgida por iniciativa propia, como reacción a la pregunta de SF que fue avalada por el profesor, es voz. Nos resulta inteligible. El estudiante presenta un relato que no había terminado de elaborar y del que no tenía suficiente certeza ("no sé si me acuerdo bien"), que fue influido por una lectura rápida de la guía ("me pareció ver") y, quizá, en particular, por la evocación de la imagen correspondiente a un ejemplo en el que se ilustra la aplicación de una homotecia de razón 2 a una región triangular (Imagen 5). SG habla de construir - a la vez que hace lo expresado- dos rectas determinadas, cada una, por lo que podríamos considerar un par de vértices correspondientes de las dos figuras; de marcar el punto de intersección de las dos rectas; luego sugiere que al deslizar de una cierta manera la figura pequeña, esta va aumentando su tamaño hasta alcanzar el de la figura grande. Enseguida, como reacción a una sugerencia del profesor respecto a considerar un tercer vértice de la figura pequeña, el estudiante traza la recta determinada por la intersección marcada antes y un tercer vértice de la figura pequeña y la extiende de manera que el tercer vértice de la figura grande también pertenezca a la recta. Termina su relato, sugiriendo que el ángulo formado por las dos rectas trazadas inicialmente es un elemento importante de un asunto que no explicita. Sin duda, también consideramos auténtica la expresión de SG. Desde el comienzo de la sesión de clase varios estudiantes han insinuado de manera vaga una relación entre homotecia y polígonos semejantes. Pero SG es el primero en sugerir, en lugar del uso de las condiciones de la definición de polígonos semejantes, una construcción relacionada con una homotecia -así no haya mencionado siquiera el término "homotecia"-; además de que lo hace en sus propias palabras (ver párrafo siguiente) independientemente de las debilidades que tiene su relato. Entrevemos como intención comunicativa de esta voz poner a consideración la idea esbozada para su elaboración como parte del discurso de la clase. 
Imagen 5. Gráfica de un ejemplo dado en la guía de lectura, cuya evocación influyó el relato de SG

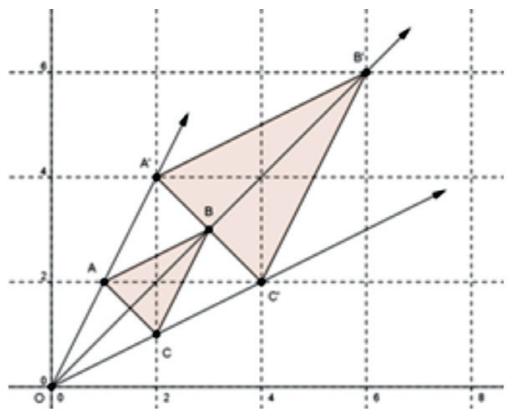

El vocabulario usado por SG no incluye términos propios del discurso geométrico, con excepción de "figura" y "línea" -usadas, respectivamente, en lugar de "polígono" y "recta"- y "ángulo". Usa "juntar" para referirse a "intersecar". Además, se vale de descripciones cuando no puede indicar aquello a lo que se refiere (e.g., "donde se juntan", "parte superior de la figura agrandada", "la misma parte de la chiquita"). Es de destacar el uso de la forma "el mismo punto en la otra figura" en vez de "puntos correspondientes", lo que pone en evidencia una confusión entre una figura que se transforma (e.g., "la figura como que se... se agrandaba de acá a acá") o dos figuras que se pueden poner en correspondencia (e.g., "la figura ya agrandada", "de la chiquita"). En suma, su lenguaje no es especializado.

Cuando el profesor le dice que pase al tablero y, así, SG dispone de las dos figuras expuestas como su mediador visual de base, los vértices que señala, las rectas que traza, la intersección que marca y señala, el arco que traza para indicar el ángulo al que se refiere y, los gestos que hace para indicar que la figura grande es transformada de la figura pequeña, todos ellos son mediadores visuales para la exposición que SG hace frente al grupo.

En la voz de SG reconocemos un relato asociado a las dos figuras del tablero, en el que menciona acciones que podrían hacer parte de un cuasi procedimiento o rutina geométrica, cuyo propósito, al parecer, sería determinar si la figura de mayor tamaño pudo ser obtenida por homotecia sobre la otra figura y, por esa vía determinar si los ángulos correspondientes tienen o no medidas iguales. No consideramos que ese relato sea una narrativa susceptible de aceptación o rechazo por no tener información suficiente.

Como reacción a la voz de SG, el profesor presenta al grupo su interpretación de la idea expuesta e inicia y sostiene un diálogo con algunos estudiantes 
tratando de obtener la respuesta a la pregunta "¿Qué debe pasar cuando yo trazo esas líneas?" que, probablemente, abriría la vía para completar colectivamente el procedimiento esbozado por SG. No tiene éxito. Vuelve a presentar su interpretación, explicitando lo que sería el criterio en términos de si se intersecan o no las tres rectas en un mismo punto, y termina afirmando que no es posible concluir necesariamente la semejanza de las dos figuras. Reconocemos en la voz de SG una contribución potencialmente productiva para el desarrollo del discurso matemático del aula, en dos sentidos. Por un lado, abordando preguntas relacionadas con las acciones mencionadas en el relato (e.g., en qué situaciones se pueden realizar, con qué propósito se realizan, cómo se usan los resultados que se van obteniendo, etc.) se podría llegar a establecer una secuencia detallada de acciones realizadas respecto a dos polígonos, que permita llegar a una narrativa relativa a los ángulos correspondientes de los polígonos en cuestión. Por otro lado, reflexionando sobre la experiencia de producir la mencionada secuencia y la secuencia misma, se puede iniciar la objetificación de tal actividad en el respectivo procedimiento geométrico.

\section{PARA TERMINAR}

En el análisis expuesto identificamos y describimos, aprovechando las herramientas del enfoque comunicacional de Sfard (2008a), voces matemáticas de cuatro estudiantes, surgidas en un intercambio verbal breve en una clase de geometría. Sintetizamos a continuación la descripción hecha.

Tres de las cuatro expresiones consideradas -la excepción es la de DG- Ilegan a ser inteligibles a través de un breve intercambio con el profesor, en el que este pide de manera verbal o gestual precisión de lo dicho y el estudiante responde pertinentemente. En los cuatro casos, se identifican expresiones auténticas surgidas y elaboradas en el curso de la conversación, lo cual se evidencia en el carácter idiosincrásico de las verbalizaciones, propio de jóvenes que están comenzando su incursión en el discurso matemático sobre polígonos semejantes.

Enfocando el vocabulario incluido en las voces y el uso de este que podemos inferir, cabe resaltar que solo en una voz aparece el término "semejante" y está empleado para preguntar sobre una propiedad de una figura con respecto a otra y no para aludir a una relación entre dos figuras. En ese sentido, el término "semejante" aún no se ha incorporado al discurso de los estudiantes explícita y cómodamente, y la relación de semejanza no se ha objetificado. 
Encontramos algunos términos especializados propios del discurso sobre polígonos semejantes: tamaño, forma, ángulo, longitud, medir longitud, razón y división cuyo uso fue analizado detenidamente. Los términos "tamaño" y "forma" se usan como atributos de una figura; sin embargo, no hay información directa sobre la significación dada a "forma"; probablemente, los estudiantes aluden con este a la apariencia captada perceptualmente de manera global, lo que puede explicar la tendencia a afirmar que dos figuras son iguales en forma, sin que medie un procedimiento distinto al escaneo visual. El término "longitud" se usa como atributo medible (posiblemente de un lado de un polígono); "razón entre dos medidas", como la división de estas; "ángulo" se usa como un objeto caracterizado por su amplitud, de manera que con frecuencia se confunde el objeto (i.e., unión de rayos con punto inicial común) con su atributo principal (i.e., amplitud). Para aludir al objeto central del tema de conversación es notorio, en todas las voces, el remplazo del término "polígono" por "figura", en lo que incurrió también el profesor.

También notamos el recurso a la descripción y al uso de deícticos para subsanar la carencia de términos especializados, hecho que, a pesar de favorecer la expresión, dificulta la inteligibilidad y genera sobreentendidos no siempre correctos; así, encontramos, por ejemplo, el uso de "parte superior de la figura ya agrandada" en lugar de "vértice $X$ ", "la misma parte superior de la chiquita" en lugar de "vértice correspondiente a X"; "se juntaban" por "se intersecaban"; "una recta puede tocar este punto" por "una recta a la que pertenece este punto", "ese con este" para indicar un lado de una de las figuras y su correspondiente en la otra.

En tres de las cuatro voces (la excepción es la de SG) reconocemos narrativas; dos de ellas objetificadas (una refiere a las figuras expuestas en el tablero, otra refiere a un par de ángulos correspondientes en las dos figuras expuestas en el tablero) y susceptibles de ser aceptadas o rechazadas; una en la que se describe un procedimiento; otra en la que se presenta una refutación sobre un procedimiento. En la voz de SG se presenta un relato de un cuasi procedimiento geométrico que incluso, nos permitimos especular, no estaba previsto en la planeación del profesor, pues no hace uso de la definición de polígonos convexos semejantes.

Las voces analizadas en este estudio nos impulsan a notar asuntos importantes para el desarrollo del discurso del aula, y también para la individualización del mismo. Sin pretensión de generalidad, mencionamos dos.

El uso apropiado de la respectiva definición para determinar si dos polígonos dados son semejantes no solo requiere tener en cuenta las condiciones que aquella impone, sino también qué se puede concluir al examinar cada una de tales condiciones. De esto nos advierte la voz de DG cuando, habiéndose concluido la 
imposibilidad de asegurar la semejanza de los cuadriláteros en cuestión por no tener la información requerida en la primera condición de la definición, enfoca la atención en lo que se podría concluir si se supiera que la segunda condición se cumple. Subyacente al uso apropiado de esta definición está el uso canónico de los conectivos lógicos y de la negación de una proposición. Esta consideración es útil para impulsar una reflexión en torno a la secuenciación de tareas para apoyar la construcción de significado de la definición de polígonos semejantes.

El uso de deícticos apoyado por el señalamiento sobre una representación gráfica es un recurso que puede ayudar considerablemente a esbozar ideas en proceso de elaboración cuando no se tiene el vocabulario especializado; y, no tener acceso a dicho recurso puede constituirse en desventaja que afecta la claridad de lo que se pretende comunicar. De esto nos advierte la expresión inicial de JH que se clarifica cuando puede señalar los ángulos de los que predica igualdad, y la de SG al proponer un cuasi procedimiento. Sin embargo, con la consideración anterior no pretendemos insinuar que el uso de deícticos sea lo deseable para participar adecuadamente en el discurso matemático del aula; sí queremos llamar la atención sobre su posible efecto para promover la participación de los estudiantes y sobre el andamiaje que se hace necesario de parte del profesor para que los estudiantes cada vez necesiten menos del recurso para poder intervenir en el discurso de la clase.

Respecto a la hipótesis inicial, voces analizadas, entre las cuales están las que presentamos aquí, nos impulsan a modificarla: en un ambiente comunicativo en el que la intervención de los estudiantes en el discurso sea sentida por ellos como segura, surgen expresiones inteligibles y auténticas, que muy probablemente son aprovechables para extender y profundizar el discurso en el aula, si el profesor además de propiciar e impulsar la intervención de los estudiantes, asume su papel de experto en el discurso para ayudarlos a que su participación sea cada vez más especializada.

\section{REFERENCIAS}

Camargo, L. (en prensa). Estrategias cualitativas de investigación en Educación Matemática. Clarke, D., Xu, L.H. y Wan, M. E. V. (2013). Spoken mathematics as an instructional strategy: The public discourse of mathematics classrooms in different countries. En B. Kaur, G. Anthony, M. Ohtani y D. Clarke (Eds.), Student voice in mathematics classroom around the world (pp. 13-32). Sense Publishers. 
Duval, R. (2017/2004). Semiosis y pensamiento humano. Registros semióticos y aprendizajes intelectuales (2a ed.) (M. Vega, Ed.). Universidad del Valle.

Esmonde, I. y Langer-Osuna, J. (2013). Power in numbers: Student participation in mathematical discussions in heterogeneous spaces. Journal for Research in Mathematics Education, 44(1), 288-315.

Forero-Sáenz, A. (2008). Interacción y discurso en la clase de matemáticas. Universitas Psychologica, 7(3), 787-805.

Gates, P. y Vistro-Yu, C. (2003). Is mathematics for all? En A. Bishop, M.A. Clements, C. Keitel, J. Kilpatrick y F. K. S. Leung (Eds.). Second international handbook of mathematics education (pp. 31-73). Springer.

Gregg, J. (1995). The tensions and contradictions of the school mathematics tradition. Journal for Research in Mathematics Education, 26(5), 442-466.

Ingram, J., Andrew, N. y Pitt, A. (2019). When students offer explanations without the teacher explicitly asking them to. Educational Studies in Mathematics, 101(1), 51-66.

Jiménez, A. Suárez, N. y Galindo, S. (2010). La comunicación: eje en la clase de matemáticas. Praxis \& Saber, 1(2), 173-202.

Jung, J. y Shütte, M. (2018). An interactionist perspective on mathematics learning: Conditions of learning opportunities in mixed-ability groups within linguistic negotiation processes. ZDM, Mathematics Education, 50(6), 1089-1099.

Kaur, B. (2013). Participation of students in content-learning classroom discourse: A study of two grade 8 mathematics classes in Singapore. En B. Kaur, G. Anthony, M. Ohtani y D. Clarke (Eds.), Student voice in mathematics classrooms around the world (pp. 65-88). Sense Publishers.

Lavie, I., Steiner, A. y Sfard, A. (2019). Routines we live by: From ritual to exploration. Educational Studies in Mathematics, 101(2), 153-176.

Ministerio de Educación Nacional (MEN) (1998). Lineamientos curriculares de matemáticas. Autor.

Morgan, C. y Sfard, A. (2016). Investigating changes in high-stakes mathematics examinations: A discursive approach. Research in Mathematics Education, 18(2), 92-119.

National Council of Teachers of Mathematics (NCTM) (1989). Curriculum and evaluation standards for school mathematics. Autor.

Radford, L. y Barwell, R. (2016). Language in mathematics education research. En Á. Gutiérrez, G. Leder y P. Boero (Eds.), The second handbook of research on the psychology of mathematics education. The journey continues (pp. 275-314). Sense Publishers.

Selden, J. y Selden, A. (1995). Unpacking the logic of mathematical statements. Educational Studies in Mathematics, 29(2), 123-151. 
Sfard, A. (2007). When the rules of discourse change, but nobody tells you: Making sense of mathematics learning from a commognitive standpoint. The Journal of the Learning Sciences, 16(4), 567-615.

Sfard, A. (2008a). Aprendizaje de las matemáticas escolares desde un enfoque comunicacional (P. Perry y L. Andrade, eds. y trads.). Universidad del Valle.

Sfard, A. (2008b). Conducción del discurso por entre la metáfora y el rigor: el análisis focal para investigar el surgimiento de los objetos matemáticos. En Aprendizaje de las matemáticas escolares desde un enfoque comunicacional (pp. 71-110). Universidad del Valle.

Sfard, A. (2008c). La cognición como comunicación: reconsiderar el aprendizaje mediado por la conversación, a través del análisis multifacético de las interacciones matemáticas de los estudiantes. En Aprendizaje de las matemáticas escolares desde un enfoque comunicacional (pp. 111-161). Universidad del Valle.

Sfard, A. (2008d). Thinking as communicating. Human development, the growth of discourses, and mathematizing. Cambridge University Press.

Sfard, A. (2008e). Aprender matemáticas como la acción de desarrollar un discurso. En Aprendizaje de las matemáticas escolares desde un enfoque comunicacional (pp. 39-63). Universidad del Valle.

Sfard, A. (2014). Why Mathematics? What Mathematics? En M. Pitici (Ed.), The best writing on mathematics 2013 (pp. 130-142). Princeton University Press.

Sfard, A. (2015). Why all this talk about talking classrooms? En L. Resnick, C. Asterhan y S. Clarke (Eds.), Socializing intelligence through academic talk and dialogue (pp. 235-243). American Educational Research Association.

Tang, S., Morgan, C. y Sfard, A. (2012). Investigating the evolution of school mathematics through the lens of examinations: Developing an analytic framework. En ICME-12 Pre-proceedings. The $12^{\text {th }}$ International Congress on Mathematical Education. Topic Study Group (pp. 5977-5986). Coex-Seoul, Korea.

Villalta, M. A. (2009). Análisis de la conversación. Una propuesta para el estudio de la interacción didáctica en sala de clase. Estudios Pedagógicos, XXXV(1), 221-238.

Wang, S. (2016). Discourse perspective of geometric thoughts. Springer Spektrum.

PATRICIA PERRY

Dirección: Kr 45A 123 64, Bogotá-Colombia

Teléfono: (57) 16470358 